\title{
Flexible optical chemical sensor platform for BTX
}

\author{
Juan Diego Arias Espinoza ${ }^{1}$, ViacheslavSazhnikov ${ }^{2}$, Sami Sabik', Dmitriy lonov ${ }^{2}$, Edsger Smits ${ }^{1}$, \\ SandeepKalathimekkad ${ }^{3}$, Geert Van Steenberge ${ }^{3}$, Michail Alfimov ${ }^{2}$, Małgorzata Pośniak ${ }^{4}$, Herman \\ Schoo ${ }^{1}$ \\ ${ }^{1}$ Holst Centre, High Tech Campus 31, 5656 AE Eindhoven, The Netherlands, \\ juan_diego.ariasespinoza@tno.nl \\ ${ }^{2}$ Photochemistry Center of the Russian Academy of Sciences, Novatorovst. 7a-1, 119421, Moscow, \\ Russia \\ ${ }^{3}$ Centre for MicroSystems Technology, ELIS Department, Ghent University-IMEC, \\ Technologiepark 914A, 9052 Ghent-Zwijnaarde, Belgium \\ ${ }^{4}$ Central Institute for Labour Protection, National Research Institute, Czerniakowska 1600-701 \\ Warsaw, Poland
}

\begin{abstract}
An in-plane flexible sensor platform for BTX detection was developed using low-cost patterning techniques and foil-based optical components. The platform was produced by a combination of laser patterning, inkjet printing and capillary filling. Key optical components such as lightguides, optical cladding layers and metallic interconnections were realized on low cost substrates such as paper and PET
\end{abstract}

The sensing mechanism is based on the change in fluorescence spectra of a reporter dye, supported over a porous matrix. Detection limits down to $1 \mathrm{ppm}$ for benzene, toluene and xylene have been measured. Response times down to a few seconds were observed for different gas concentrations.

Key words: BTX, optochemical sensor, flexible sensor, fluorescence, polymer lightguides, inkjet printing

\section{Introduction}

Benzene, toluene and xylenes (BTX) are important air pollutants which show a high potential hazard to human health even at trace amounts, especially benzene due to its carcinogenicity. Although a number of sensors, capable of BTX detection, have been demonstrated [1-5] they are sensitive to other volatile organic compounds (VOCs) and are poor at identifying chemically and structurally similar BTX. The only way to detect these compounds separately is to utilize gas chromatography [6] or a selective preconcentration technique [7]. Thus development of a selective and highly sensitive sensor for BTX detection is still an important and a challenging task.

Optochemical sensors involving a diverse scheme of sensing strategies have been employed in detecting presence and concentration of a wide range of analytes and have applications as diverse as medical and biochemical fields to security systems and food- packaging industry ${ }^{1}$. They are used for gas detection, either using direct or reagent mediated methods. Direct methods require the measurement of an intrinsic optical property of the analyte, like absorption or luminescence. In reagent-mediated or indirect methods, the change in the optical response of an intermediate agent, usually an analyte-sensitive dye molecule is used to monitor analyte concentration.

Fluorescence detection is one of the prominent optical techniques used for optochemical sensors. Fluorescence sensing can provide ultra-high sensitivity, fast response times, the capability of continuous measurements and requires a minimal amount of analyte.

Besides the physical or chemical process involved in the sensor transduction, the success of a sensor depends on the nature of the physical platform on which it is based[8]. Consideration of the transduction mechanism dictates the design of the optical platform, in 
order to obtain high efficiency in light delivery and collection.

A novel in-plane flexible sensor concept is proposed using foil-based optical technology and low-cost patterning techniques. The sensor makes use of the changes in the fluorescent spectra by exciplex formation of dibenzoylmethanoborondifluoride $\left(\mathrm{DBMBF}_{2}\right)$ and BTX. The principle of transduction of this dye renders the platform selective for the targeted gases, with a fast and reversible response.

The complete sensor system consists of lightguides, excitation light source, photodetectors, sensing layer and coupling structures, that in a whole are integrated in a flexible electronic platform.

\section{BTX Sensing principle}

When a dye molecule absorbs light, its electronic properties change dramatically, and may participate in reactions not observed in non-excited state[9]. A particular case is the formation of complexes with molecules of different structure, called exciplexes.

The formation of these exciplexes modifies the fluorescence emission of the uncomplexed dye in two ways: by quenching or reduction of the original fluorescence and by the appearance of a new fluorescence band at a longer wavelength.

The wavelength of this new fluorescence band is dependent on the molecule involved in the complex with the dye[10], a factor that can be used to identify and detect analytes.

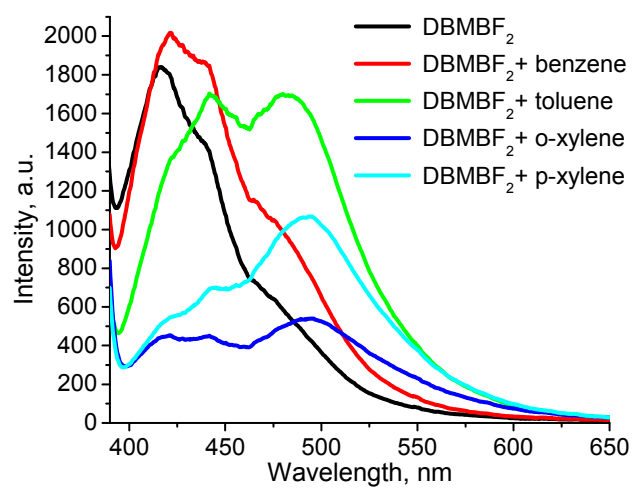

Figure 1: The fluorescence spectrum of a developed material (black). Changes in fluorescence spectrum of the material in the presence of saturated vapors of benzene (red), toluene (green), o-xylene (dark blue) and $p$-xylene (blue)

\section{Detection of monoaromatics with $\mathrm{DBMBF}_{2}$}

The formation of exciplexes between $\mathrm{DBMBF}_{2}$ and alkylbenzeneshas been studied thoroughly [10-12] and sensors materials based on the fluorescence of this complex have been suggested[13]

Thepositions of the exciplex fluorescence maxima [14]depend on the ionization potential of the donor (BTX molecule) and electron affinity of the acceptor $\left(\mathrm{DBMBF}_{2}\right)$.

Figure 1shows the changes in the fluorescence spectra of the $\mathrm{DBMBF}_{2}$ dye upon exposure to saturated $B T X$ vapors.

Sensitivity of $\mathrm{DBMBF}_{2}$ in a porous and polymeric matrix

To evaluate the fluorescent response of the dye, it was deposited over a hydrophobic porous support. The intensity of the emission of the exciplexes of the different gases was monitored. Figure 2 shows the fluorescent changes for different concentrations of toluene.

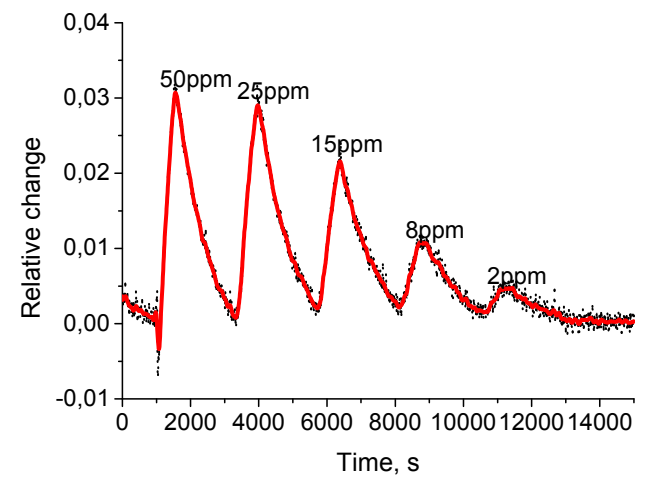

Figure 2: Toluene response curve of $\mathrm{DBMBF}_{2}$ supported over porous matrix

Similar measurements were carried out for benzene and isomers of xylene, and the lowest detected concentrations and corresponding response times for these concentrations are summarized in Table 1.

Table 1: Lowest detected concentrations(LDC) and response time of $D B M B F_{2}$ over porous support

\begin{tabular}{|c|c|c|}
\hline Gas & LDC / ppm & $\begin{array}{c}\text { Response time } \\
\text { /s }\end{array}$ \\
\hline Benzene & $16,6^{*}$ & 300 \\
\hline Toluene & 2 & 47 \\
\hline m-Xylene & $2^{*}$ & 67 \\
\hline o-Xylene & $0,8^{*}$ & 64 \\
\hline p-Xylene & $0,5^{*}$ & 67 \\
\hline
\end{tabular}

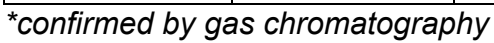


Different polymer-nanoparticles composites formulations were also prepared in order to enhance the sensitivity/selectivity of the system. The fluorescent response of the dye dispersed in these composites was evaluated between different formulations, in order to obtain the most suitable for gas sensing properties. The base polymers for these composites were aliphatic acrylates of different chain length. An example of the fluorescent response of two different composites is shown inFigure 3.

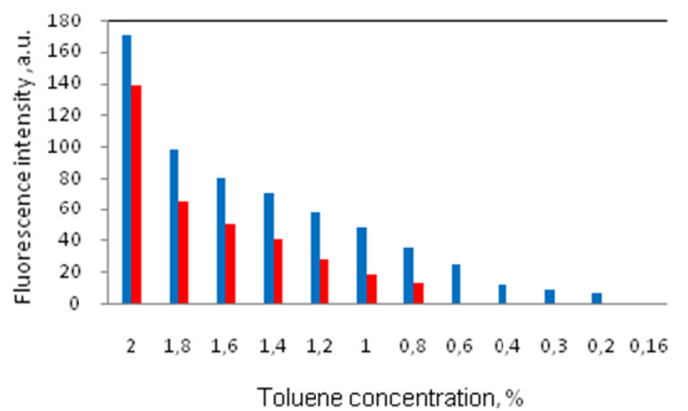

Figure 3: Fluorescent response of two composites based on acrylic polymers. Butyl acrylate based composite (blue) and lauryl acrylate composite (red)

\section{Reversibility of sensor response}

The formation of exciplexesbetween DBMBF2 and BTX's is a reversible process[15], [16].This allows the sensor to be used in multiple or continuous measurement sensors.

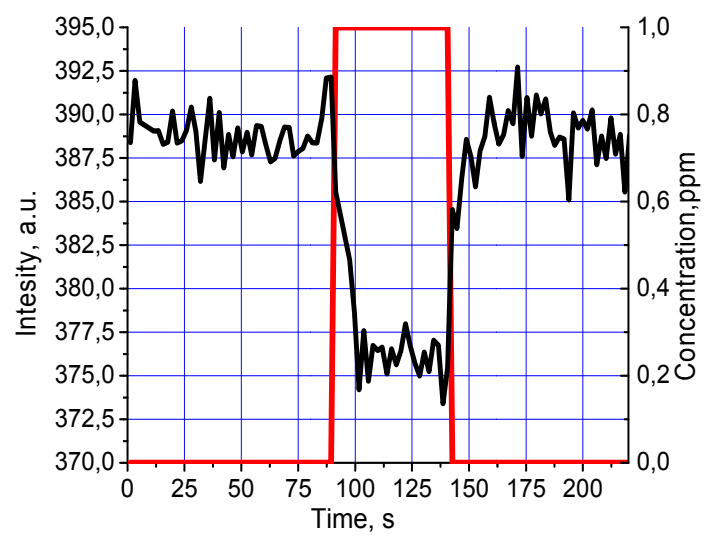

Figure 4: Dynamic fluorescence response of a sensor material to $p$-xylene vapor with a concentration of $1 \mathrm{ppm}$. The red line represents inlet and outlet of $p$-xylene vapor

\section{Optical platform architecture}

Recent advances in modern optics and electronics hasdriven the miniaturization of optical platforms, without sacrificing on the performance of the components or inflating their costs.
Together with new techniques for patterning of flexible electronics, we haddevelopedfoil-based in-plane optical sensor platforms.

In the first one, electric interconnections and conducting pads were created over on PET substrates with a combination of screen printing and laser patterning techniques.

The lightguides were obtained by capillary filling of a PDMS mold and plasma bonding to the substrate. The mold itself acts as a cladding layer in this system.
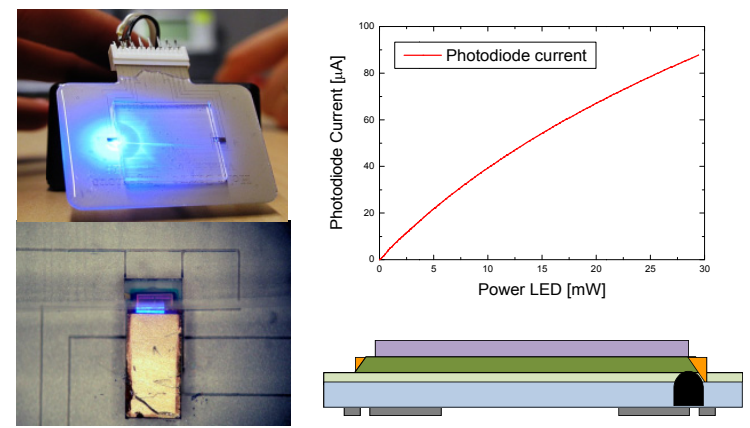

Figure 5: a) Laser ablated, mold filled optical platform. b) Detail of outcouplingmicromirror over diode. c) Photodiode response,d) Diagram of platform: Sensing layen $\square$, Lightguide core $\square$, Cladding $\square$, Substrate $\square$, Silver interconnections $\square$, Mirrors $\nabla$, LED $\cap$, Photodiode

The second platform is based on inkjet printing of the same materials (metallic interconnections, light guide core and clad), using paper and pen as substrates.
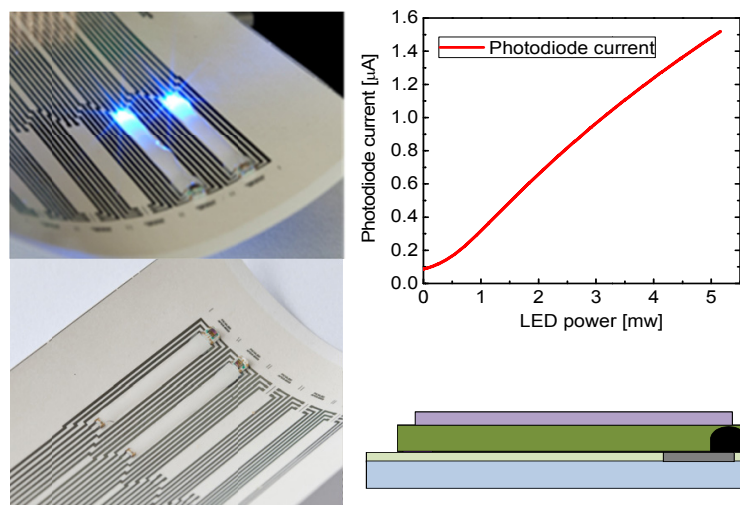

Figure 6: a,b) All inkjet printed optical platform over a paper substrate.c)Photodiode response,d) Diagram of platform: Sensing layer $\square$, lightguide core $\square$, cladding $\square$, substrate $\square$, silver interconnections $\square$, $\angle E D$, photodiode

\section{Conclusions and future work}

We have demonstrated the sensing principle for detection of $\mathrm{BTX}$ gases by using $\mathrm{DBMBF}_{2}$ as fluorescent dye. Its integration in solid supports and sensing properties were successfully evaluated. 
Two foil based, flexible optical platforms were developed and tested.Integration of the different optical components and the patterning of its layer was successfully accomplished

A sensing layer, comprising of $\mathrm{DBMBF}_{2}$ dispersed in a composite matrix, will be deposited over the lightguides core in the next stage of the project. Fluorescent enhancement metal layers will be studied to improve sensitivity of the sensor.

\section{Acknowledgements}

This work is conducted in the framework of the FP7 European Project "Ingenious" (NMP-20091.2-3: Nanotechnologies, coordinated call with Russia, A: Optical chemical sensing, grant agreement number 248236).

\section{References}

[1] MR Almasian, N Na, F Wen, S Zhang, X Zhang. Development of a plasma-assisted cataluminescence system for benzene, toluene, ethylbenzene, and xylenes analysis, Analytical chemistry 82, 3457-3459 (2010); doi: 10.1021/ac1006975.

[2] GS Aluri, A Motayed, AV Davydov, VP Oleshko, $\mathrm{K}$ a Bertness, $\mathrm{N}$ a Sanford, MV Rao. Highly selective GaN-nanowire/TiO(2)-nanocluster hybrid sensors for detection of benzene and related environment pollutants., Nanotechnology 22, 295503 (2011); doi: 10.1088/0957-4484/22/29/295503.

[3] R Kadir, A Yimit, H Ablat, M Mahmut, K Itoh. Optical waveguide BTX gas sensor based on polyacrylate resin thin film., Environmental science \& technology 43, 5113-6 (2009); doi: 10.1016/j.aca.2009.10.056.

[4] Q Qi, T Zhang, L Liu, X Zheng. Synthesis and toluene sensing properties of $\mathrm{SnO} 2$ nanofibers, Sensors and Actuators B: Chemical 137, 471475 (2009); doi: 10.1016/j.snb.2008.11.042.

[5] M Mori, Y Sadaoka. Potentiometric VOC detection at sub-ppm levels based on YSZ electrolyte and platinum electrode covered with gold, Sensors and Actuators B: Chemical 146, 46-52 (2010); doi: 10.1016/j.snb.2010.02.001.

[6] LIB Silva, FDP Ferreira, T a P Rocha-Santos, a $C$ Duarte. Carbon nanotube field-effect transistor detector associated to gas chromatography for speciation of benzene, toluene, ethylbenzene, (o-, m- and p-)xylene., Journal of chromatography. A 1216, 6517-21 (2009); doi: 10.1016/j.chroma.2009.07.060.

[7] Y Ueno, A Tate, O Niwa, H-S Zhou, T Yamada, I Honma. High benzene selectivity of mesoporous silicate for BTX gas sensing microfluidic devices., Analytical and bioanalytical chemistry 382, 804-9 (2005); doi: 10.1007/s00216-004-2974-6.

[8] C McDonagh, CS Burke, BD MacCraith. Optical chemical sensors., Chemical reviews 108, 40022 (2008); doi: 10.1021/cr068102g.

[9] Demchenko, A.P. Introduction to fluorescence sensing. Springer, 2008, p.571.

[10] $\mathrm{YL}$ Chow, $\mathrm{Cl}$ Johansson. Exciplexes of (Dibenzoylmethanato)boron/Benzenes: The Control of Exciplex Electronic Structure, Journal of Physical Chemistry 99, 17558-17565 (1995); doi: 10.1021/j100049a015.

[11] YL Chow, Z-L Liu, Cl Johansson, Jichilshiyama. The Structure and Decay Dynamics of Exciplexes Derived from DibenzoylmethanatoboronDifluoride and Alkylbenzenes in Cyclohexane, Chemistry $A$ European Journal 6, 2942-2947 (2000); doi: 10.1002/1521-3765(20000818)6:16<2942::AIDCHEM2942>3.0.CO;2-\#.

[12] YL Chow, S-S Wang, Cl Johansson, Z-L Liu. Mechanistic Studies on the Dual Reaction Pathways of Singlet Excited Dibenzoyl(methanato)boron Difluoride (DBMBF2): Reactions of the Excimer and Exciplexes, Journal of the American Chemical Society 118, 11725-11732 (1996); doi: $10.1021 / \mathrm{ja} 9610444$.

[13] VA Sazhnikov, VM Aristarkhov, AG Mirochnik, EV Fedorenko, MV Alfimov. Fluorescence quenching of silica gel-adsorbed (Dibenzoylmethanato)boron difluoride by polar solvent vapor, Doklady Physical Chemistry 437, 35-37 (2011); doi: $10.1134 / \mathrm{S} 0012501611030031$

[14] H Knibbe, D Rehm, A Weller. Bildung von Molekülkomplexen im angeregten Zustand; Zusammenhang zwischen Emissionsmaximum und Reduktionspotential des Elektronakzeptors, Zeitschrift für Physikalische Chemie 56, 95-98 (1967); doi: 10.1524/zpch.1967.56.1_2.095.

[15] YL Chow, Cl Johansson, Z-L Liu. Ground- and Excited-State Electron Donor-Acceptor (EDA) Complexes from (Dibenzoylmethanato)boron Difluoride and Substituted Benzenes: Their Relation to the Reaction Mechanism, The Journal of Physical Chemistry 100, 1338113385 (1996); doi: 10.1021/jp961000h.

[16] G Orellana. Fluorescence-based sensors, Optical chemical sensors 224, 99-116 (2006); doi: 10.1007/1-4020-4611-1_6. 